\title{
Feedback: A Synopsis of Readers' Responses
}

\section{A five stage model of management evolution}

The rationale for advancing a staged model of management evolution is to bridge human and economic dimensions of management. Effective management is the product of both human and economic achievements and developments over time in a given environment, organization, society and culture.

The concept of evolution is chosen because of the loaded meanings other given to other possible terms like growth and development. Evolution implies orderly change and the accumulation of characteristics over time. It is the opposite of revolution or radical and erratic change. Growth and development also have special meanings. in an economic sense which may not be equally applicable in a human sense. Hence, the preference for the concept of evolution to describe management stages and shifts.

Researchers in management recognize the problems and pitfalls of trying to present a universally comprehensive model of something as diverse as management. Yet, after several decades there is still too much fragementation of knowledge about management. Only recently has it become popular to relate environmental influences and technology to organizational choices about structures, strategies and systems in an open systems context. There is still considerable division about contributions of scientific management and management science, and general systems theory and systems science to the field of management. Also how do these developments relate to, and differ from, the current focus on strategic management systems? Hopefully a stage model can help bridge differences in our understanding of what role science, systems and strategies play in management thinking and practice.

In particular, there is a need for more consistent operational definitions of basic management con- cepts. For this reason, concept relevant to symbolic, static, scientific, systemic and strategic management are advanced. These terms, as presented, provide a synthesis of the key elements common to a particular era of management thinking and approach to management. They go beyond any one writer's definition. Also, each term should be viewed in relative sense rather than as absolute measures. There are varying degrees of emphasis on using symbolism, static methods, scientific inquiries, systemic interactions, and strategic concerns by managers in even the same organization and work environment. This enhances and adds to the richness of individual managerial styles and differences.

In fact, the purpose of this article is to promote continuing dialogue and discussions by various researchers and specialists to advance additional classificatory, descriptive and prescriptive models of management. Out of greater interaction between disciplines can emerge more meaningful, complete an objective models of management in the future. You are invited to contribute your ideas to this undertaking by writing the author. Together we can improve our understanding of management and bridge theory and practice. Evaluate the model presented in terms of all types of human activities you are associated with. How applicable are the stages in describing particular work environments, organizations, societies and cultures?

Human Systems Management is a logical medium to assume a leadership role in achieving continuing dialogue in these areas of concern to managers and leaders everywhere.

August W. SMITH 


\section{Macro-marketing: does it exist at all?}

In the last decade an increasing number of articles have been published on a concept called macro-marketing. The majority of the papers deal with marketing used at a macro-level, e.g. as a change agent for governmental bodies, and only a few papers treat the macro-effects of micro-marketing.

The former is, in my opinion, a contradiction. Traditional micro-marketing has been developed in the western capitalistic societies and is based upon the beliefs of the liberal society and the protestant model of man (two inseparable beliefs). Using traditional marketing models and methods at a macro-level implies a contradiction, because you cannot at the same time believe in "the invisible hand of Adam Smith" and the free will of the individual, and use models based on these beliefs to change the "unchangeable". Normative macro-marketing will therefore inevitably lead to a confirmation of the market economy.
The latter, macro-marketing being the "undirected synergistic effect created by the many interdependent exchange processes on the micro-level" can lead to very interesting results with regard to macro-marketing; you are not tied up with the liberal/ protestant paradigms anymore. At the micro-level, you have the choice between consumer and producer sovereignty as a basis for analyzing the tasks of marketing. And 'adding up' these two views at the macrolevel, you either get the results that marketing is the change agent within the limits of the liberal society, or you reach the conclusion that marketing is partly responsible for the present society, with its goods and bads. Marketing, thereby, can be blamed for overconsumption, energy crisis, alienation, etc, and cannot be considered value-free, as in the consumer dominated society.

Sфren HEEDE 